\title{
MULTISPECTRAL IMAGE SEGMENTATION BASED ON CARTESIAN COMPLEXES AND THEIR ASSOCIATED ORIENTED MATROIDS
}

\author{
J. A. Valero ${ }^{\text {a }}$, I. Lizarazo $^{\text {b }}$, P. A. Arbeláez ${ }^{\text {c }}$ \\ ${ }^{a}$ Facultad de Ingeniería, Universidad Distrital, Bogotá, Colombia - jvalero@ udistrital.edu.co \\ ${ }^{\mathrm{b}}$ Facultad de Ciencias Agrarias, Universidad Nacional de Colombia, Bogotá, Colombia - ializarazos@ unal.edu.co \\ ${ }^{\mathrm{c}}$ Universidad de los Andes, Bogotá, Colombia - pa.arbelaez@uniandes.edu.co
}

KEY WORDS: Image Segmentation, Locally Finite Spaces, Matroids, Cartesian Complexes

\begin{abstract}
:
A number of strategies have been used to include spatial and topological properties in the image segmentation stage. It is generally accepted that grouping of nearby pixels by modelling neighbourhood relationships as $(a, b)$ connected graphs may lead to meaningful image objects. In such approach, however, topological concepts may suffer from ambiguity since image elements (pixels) are two dimensional entities. This paper evaluates whether an alternative representation of digital images based both on Cartesian complexes and oriented matroids may improve multispectral image segmentation by enforcing topological and geometric properties and then be used in the classification stage. A conceptual model is defined, using Cartesian complexes, in order to link combinatorial properties of axiomatic locally finite spaces and their associated oriented matroids for involving topological properties. The proposed approach uses a layered architecture going from a physical level, going next through logical geospatial abstraction level and then through the Cartesian complex logical level. Additionally, there is a layer of oriented matroids composed by conceptual elements in terms of combinatorics for encoding relevant features to multispectral image segmentation. First, it is conducted an edge detection task, next an probability contour map using a Cartesian complex space rather than the conventional image space and finally, an image classification using random forest method. A computational solution including several components was developed using a framework for parallel computing. The performance of this solution was assessed using a small subset of GEOBIA2016 benchmark dataset. It is shown that the usage of a partial implementation of Cartesian complexes and associated oriented matroids is computationally but does not increase classification accuracy.
\end{abstract}

\section{INTRODUCTION}

A number of strategies have been used to include spatial and topological properties in the image segmentation stage. The most common practice for grouping nearby pixels is to model neighbourhood relationships as (a, b) connected graphs as it has been accepted that it may lead to meaningful image objects. In this approach, however, topological concepts may suffer from ambiguity since image elements (pixels) are two dimensional entities composing a spatially uniform grid cell in which there are not uni-dimensional nor zero-dimensional elements to build boundaries. This paper at establishing to stablish whether an alternative representation of digital images based both on Cartesian complexes (Kovalevsky, 2005) and oriented matroids (Whitney, 1935)(Oxley, 2006) may improve multispectral image segmentation by enforcing topological and geometric properties. A conceptual model is defined, using axiomatic locally finite spaces (ALFS) provided by Cartesian complexes, in order to link ALFS combinatorial properties and their associated oriented matroids in order to take into account topological properties.

The proposed approach uses a Cartesian complex layered architecture (CCLA) (Figure 1) going from a physical level represented by the multispectral image data set, and then passing through the logical geospatial abstraction level (GDAL) that allows manipulation of the underlying physical level. This abstraction level supports Cartesian complex logical level which provides required topological and geometric elements. Those elements allow for conducting first an edge detection task and then a hierarchical watershed using a Cartesian complex space rather than the conventional uniform grid cell. Additionally, there is a layer of oriented matroids composed by conceptual elements in terms of combinatorics for encoding relevant features to calculate oriented deriva- tive filters and oriented texture gradients that builds on (Arbelaez et al., 2011) but this time using a Cartesian complex space.

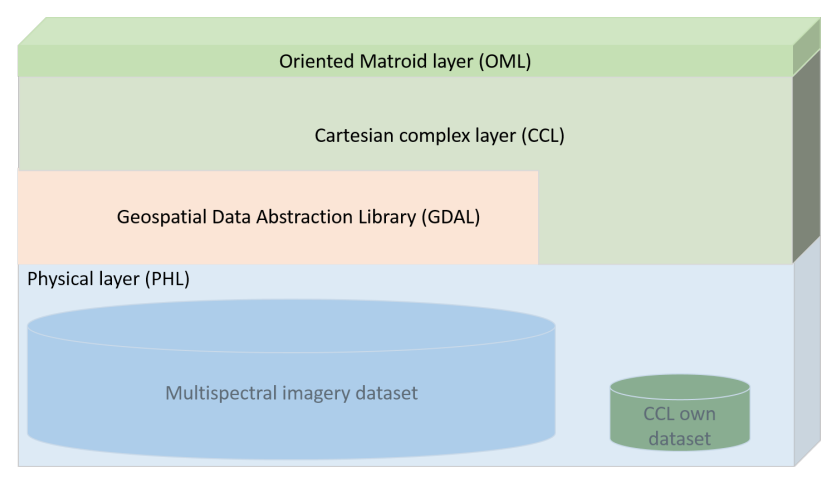

Figure 1. Multispectral image segmentation conceptual model

The complete implementation includes several components: (i) Cartesian complex core (CCC), on top of the GDAL implements a topological and geometrical space: (ii) Region boundary encoding component (RBE) provides a block complexes (BC) boundary encoding: (iii) Directional filtering (DF) supports texture detection based on filter banks. (iv) Orient gradient (OG) implements the calculation, based on oriented matroids, required to produce the maximum gradient image: (iv) Boundary cell affinity (BCA) supports the affinity analysis between cracks on detected digital straight line segments: (v) Ultrametric contour map (UCM), using mathematical morphology on a point and crack graph, provides means to produce the final Cartesian complex segmentation. 
For this study, a CCLA computational implementation (CCLAI) using only CCC, GDAL, DF and OG components was developed applying the Fork/Join framework for parallel computing. The performance of this partial solution was assessed using a small subset of GEOBIA2016 benchmark dataset. The segmented image was classified using random forest (RF) method (Tso and Mather, 2009).

This article is organized as follows. Section 2 discusses first the two most common topological ambiguities found in a conventional pixel space representation and and describes the dataset used. Descriptions of boundary strength, watershed transform and classification methods used in the experiments are also included. Results and discussion are presented in Sections 3 and 4 respectively and finally, main conclusions are drawn in Section ??.

\section{DATA AND METHODS}

\subsection{Conventional space representation ambiguities}

In the conventional representation of images, i.e. a tessellated space, topological concepts may suffer from ambiguity since image elements (pixels) are two dimensional entities. This inaccuracy is present in representations of images such as 4-connected, 8-connected and (a,b)-connected graphs (Rosenfeld, 1970) (Kong and Rosenfeld, 1991).

Figure 2(a) shows on the left, in a continuous space, how withdrawing points from the boundary between the interior and exterior sets - previously disconnected by a Jordan curve- these sets become connected and on the right how boundaries of the interior and exterior sets, are one-dimensional and coincide perfectly (red line).
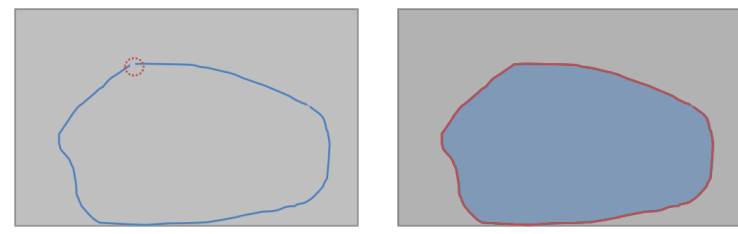

(a) Continuous space

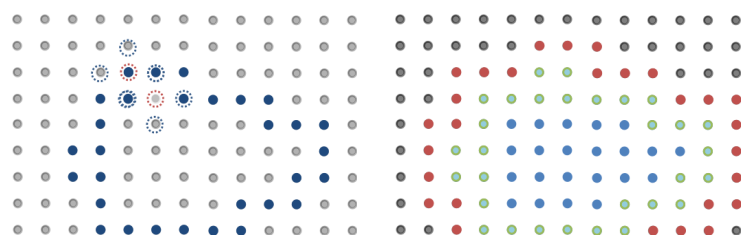

(b) Conventional digital space

Figure 2. Connectedness and Boundary definition

Figure 2(b) shows on the left,in a conventional digital space and using the 4-connected criterion, how by removing an element (encircled in red) from the boundary (depicted using blue points), the corresponding interior and exterior sets do not become connected. Actually, in the conventional digital space, it is not possible either to establish unambiguously such a connectivity using the 8-connected criterion. In this case, the two sets remain always connected even without removing the element under consideration from the boundary. The right of this figure shows how each of the two sets have different boundary (exterior's boundary is red and interior's boundary is green), and that both boundaries are two-dimensional (all the elements comprising this space are two-dimensional). These paradoxes may be particularly significant for image segmentation and classification when considering topological relationships such as neighbourhood, contiguity, connectivity, and boundary.

Digital image processing based on representations of space that do not meet the topological axiomatic postulates (Munkres, 1999) may cause geometric algorithms to be affected by paradoxes that lead to ambiguous or erroneous decisions. While it is commonly accepted that a raster image matches human perception to some degree, it seems that several important concepts for image analysis, such as the connectivity of regions, their boundaries, and the adjacency between them, are not explicitly present. This lack of topological awareness of grid-based image representation is clearly an important limitation for image analysis in computer environments (Kovalevsky, 1989).

On the other hand, it is well known that algorithms based on geometric characteristics have high complexity since the number of possible situations to be considered increases significantly with the number of dimensions of the objects under evaluation (Worboys and Duckham, 2004). Additionally, another problem that arises during the implementation phase of geometric algorithms is the impossibility of having an accurate arithmetic in terms of real numbers. This leads to resort to an accurate arithmetic in terms of integer or rational numbers that decreases performance (de Berg et al., 2008).

To sum up, conventional methods for multispectral image segmentation are based on digital structures that violate well-established topological axioms and geometric algorithms that assume a continuous space computing model. This study explores an alternative approach to address such a problem.

\subsection{Data}

For the boundary reliability assessment Berkeley Segmentation Data Set and Benchmarks 500 (BSDS500) (Martin et al., 2001) was used. A training dataset with two hundred natural images along their ground truth segmentations were used for producing the precision and recall curves. The data set used in the segmentation and classification experiments are further described.

The International Society for Photogrammetry and Remote Sensing (ISPRS) has launched the "semantic labeling contest" and provided airborne image datasets consisting of very high resolution true ortho photo (TOP) tiles and corresponding digital surface models (DSMs) of Vaihingen, a small village area in Germany, derived from dense image matching techniques.

The TOP are 8 bit TIFF files with three bands: near infrared, red and green bands delivered by the camera. The DSM are TIFF files with one band; the grey levels (corresponding to the DSM heights) are encoded as 32 bit float values. Images cover urban scenes that have been classified manually into five land cover classes: Building, Low vegetation, Tree, Car and Impervious surfaces.

In this study, it was selected tile 11 of Vaihingen data set. Two non-overlapping 1000 X 1000 pixel windows, one from $(400,400)$ to $(1400,1400)$ for the training stage and the other one from $(893,1400)$ to $(1893,2400)$ were subset from tile 11 for the final classification and assessment stages. A whole tile was not used because available computations resources were not good enough to work with images of that size in all conducted in the experiments. 


\subsection{Boundary strength and watershed transform}

Previous work on topology and geometry preserving image segmentation includes definition of a predicate for measuring evidence for a boundary between two regions using a graph-based representation (Felzenszwalb and Huttenlocher, 2004); abstract cell complexes for implementing topological relations needed to perform unambiguously segmentation of digital images in computer vision (Kovalevsky, 2005); image objects generation using the fractal network evolution (FNEA) (Yu et al., 2006); multiscale image segmentation approach in which urban land use is divided into different levels forming a hierarchical network structure (Kong et al., 2006); hybrid split-merge method for image segmentation based on computational geometry and topology using persistent homology (Letscher and Fritts, 2007); objectoriented spectral and textural classification algorithm for producing a map of structural classes after a textural analysis (Johansen et al., 2007).

Previous work also includes an active image segmentation method that distorts the image using B-Spline free-form deformations in order to match initial outlines and segments multiple objects simultaneously (Li and Sun, 2010), unified approach to contour detection and image segmentation using first an Oriented Watershed Transform (OWT) to build a hierarchical segmentation and then, from an initial RAG, a hierarchy formed based on the links weights as a measure of dissimilarity between regions (Arbelaez et al., 2011), a method for integrating topological image properties within a random field image segmentation model, which does not pose topological restrictions in the energy minimization stage (Chen et al., 2011) and a bottom-up strategy improving the agglomeration using more information besides boundary. Using supervised machine learning techniques it is possible to predict when two super pixels should be merged (Nunez-Iglesias et al., 2013).

In this study the first experiment includes a preliminary boundary reliability assessment in order to ensure CCLAI is good enough for producing boundary definitions. The assessment consisted in a gradient magnitude stage followed by a watershed production stage in both CCLAI and Matlab software. In the first stage, a gray scale image was computed from the original datasets (using Equation 1) by a three band lineal combination which was $x$ and $y$ sobel (Szeliski, 2010) filtered for finally calculating the gradient magnitude (using Equation 2).

$$
\text { grayimage }=\frac{1}{3}\left(b_{1}+b_{2}+b_{3}\right)
$$

where

$$
b_{i}=i \text { image band }
$$

$$
\text { gradientmagnitud }=\sqrt{\text { sobel }_{x}^{2}+\text { sobel }_{y}{ }^{2}}
$$

where $\quad$ sobel $_{x}=$ horizontal sobel filter

$$
\begin{aligned}
& \text { sobel }_{x}=\text { horizontal sobel filter } \\
& \text { sobel }_{y}=\text { vertical sobel filter }
\end{aligned}
$$

As CCLAI has available interpixel elements, it was used a $2 \times 3$ sobel fiter for horizontal edgels ( 1 -cells) and a $3 \times 2$ for verticals in contrast with the two $3 \times 3$ sobel filters used in Matlab. Raw gradient magnitude images where thinned using non-maximum suppression (Canny, 1986). The assessment methodology defined in (Arbelaez et al., 2011) was applied on the BSD500 images.

In the other experiment previously computed gradient magnitude images were introduced as watershed hierarchy production inputs. In this case the non maximum suppression procedure was not applied as the watershed transform should obtain boundary definitions. The watershed production implementation was based on the algorithm proposed in (Vincent and Soille, 1991) modified to use edgels (1-cells) available in the Cartesian complex space.

For the watershed hierarchy production a hierarchical grouping based on watershed ids with two to seven number of clusters was done. This ids were used as a watershed id produced by CCLAI framework is a poset in which the partial order relationship is given by the flooding process implicit in (Vincent and Soille, 1991) algorithm. It was necessary to match Matlab watershed ids to this order by a relabelling procedure.

On the architecture mentioned in section 1 the watershed production process shown on Figure 3 was implemented and used for obtaining classification superpixel datasets.

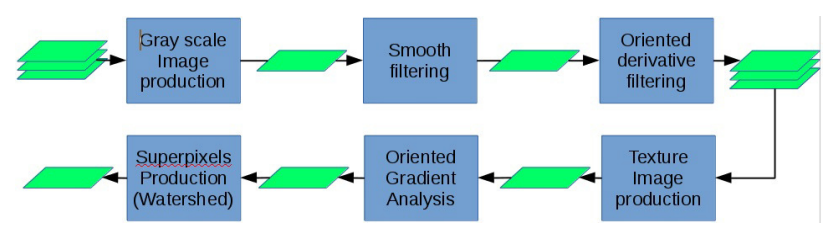

Figure 3. Superpixel production pipe line

First, a gray scale image was computed from the original datasets and then it was Gaussian filtered. Next an oriented derivative filter bank with seven orientations was applied from which a texture image was produced by a k-means grouping (Kanungo et al., 2002). Then, an oriented gradient image was calculated from the so produced texture (Leung and Malik, 1996) image taking the maximum for each of seven orientations. Finally, the watershed algorithm CCLAI implementation was applied to obtain the superpixel images.

Also, training and testing texture images were produced for the classification stage. For that, as can be seen on Figure 4 first a gray scale image was computed from the original datasets over which an oriented Gaussian filter bank with three orientations and three scales was applied in order to generate the edgel (1cell) texton response image. The number of filters in the bank was 21 - 3 even-symmetric (Gaussian second derivative), 3 oddsymmetric (Gaussian second derivative with Hilbert transform) and one center-surround all of them at each one of three scales but as each pixel has two own edgels (Kovalevsky, 2005) the final number of response texton channels were 42. Finally, "texture" images were obtained by k-means grouping with 64 clusters applying training texture image cluster centres to texton test image to obtain the testing texture image. Each texture image has two channels one for vertical edgels and one for horizontal ones.

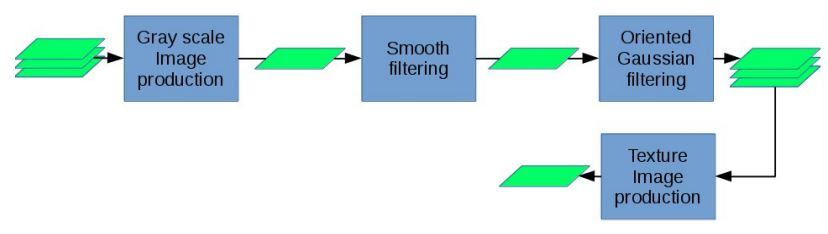

Figure 4. Superpixel production pipe line

All algorithms were implemented on the underlying Cartesian complex 2-dimensional space using specifically edgels (1-cells) interpixel space elements instead of the pixels available in a conventional digital space. Hyperplane central arrangements from 
the Oriented Matroid Layer (OML) were used to compute oriented derivative filtering responses. Fourteen histogram sectors used in oriented gradients calculation were encoded by using OML layer to compute combinatorially the final gradient image. The edgel gradient value was calculate as in (Arbelaez et al., 2011). The resulting over segmented image was used for classification describing each superpixel with multispectral, "texture" (k-means grouping), normalized difference vegetation index (NDVI) (calculated using Equation 3) and Digital Surface Model (DSM) mean responses inside it. The classification stage was conducted as described in Section 2.4.

$$
N V D I=\frac{b_{1}-b_{2}}{b_{1}+b_{2}}
$$

where

$$
\begin{aligned}
& b_{1}=\text { near infrared band } \\
& b_{2}=\text { red band of the image }
\end{aligned}
$$

\subsection{Classification procedure and assessment of classifications}

Three classification scenarios were defined. In the first, shown on Figure 5, the selected window was classified taking into account only the three spectral bands available for both the original pixel image and super pixel image produced by watershed transform previously computed.

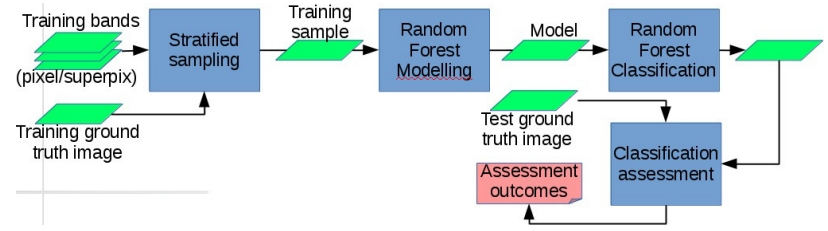

Figure 5. Spectral bands scenario

In the second scenario shown on Figure 6 the selected window was classified taking into account besides three spectral bands those corresponding to texture channels for both original pixels and super pixels.

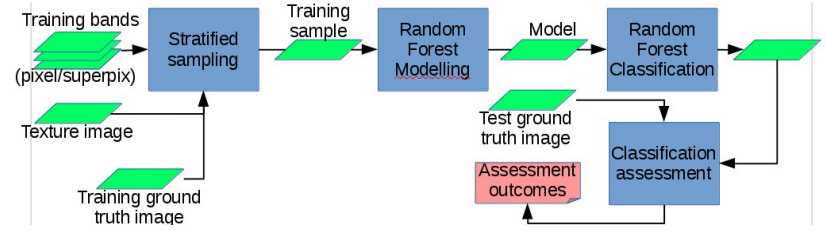

Figure 6. Spectral bands and texture scenario

In the third scenario shown on Figure 7 the selected window was classified taking into account besides three spectral bands and those corresponding to the texture channels, channels corresponding to NDVI and DSM for both original pixels as super pixels. At the end six random forest classification were run.

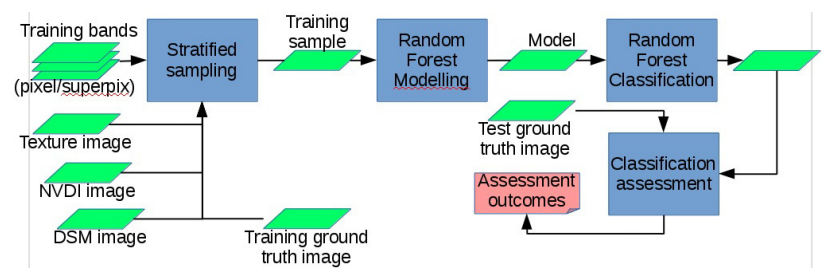

Figure 7. Spectral bands, texture, NDVI and DSM scenario
An overview of general steps of image classifications and its assessment is described in the following.

1. Training: A set of 3000 observation points was extracted from training window. A stratified sampling procedure was used in order to guarantee the same category rate in the sample as in the training window. The same set of observation points was used in all six classifications.

2. Extracting spectral signatures: the next step computed statistics from radiometric values for each class based on training sample feature space for each one of the classification scenarios previously described. Then, the model with the characteristic patterns of each class was created training each random forest classification model with 50 trees. The same model was used for both pixel and superpixel images.

3. Image classification: the image classification was carried out by random forest technique applying the respective mode generated in the previous step to the selected window. All three scenarios for both pixel and superpixel were considered to producing six random forest classifications.

4. Thematic accuracy evaluation: this step used the whole selected window reference classification (one million pixels) as validation data set. A normalized confusion matrix, the classification overall accuracy and the precision and recall were calculated for each classification outcome.

Reference classification images are shown on Figure 8. Blue areas correspond to building category, cyan to Low vegetation, green to Tree, yellow to Car and white to Impervious surfaces.

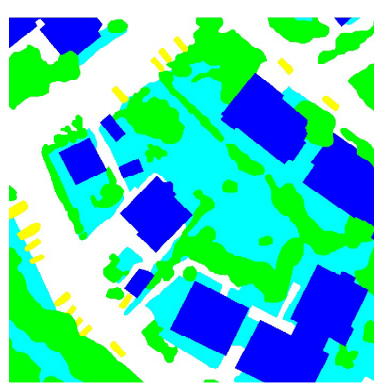

(a) Training

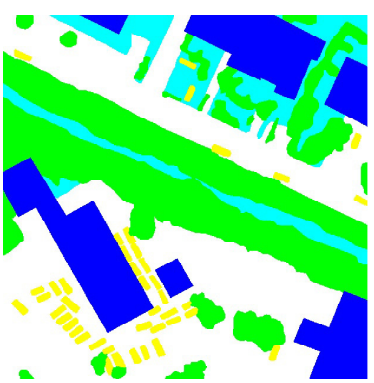

(b) Test
Figure 8. Reference classification images

\section{RESULTS}

\subsection{Segmentation}

Figure 9(a) shows a comparative boundary strength precision and recall assessment between this work outcome (green line) and conventional Matlab watershed implementation (red line) against BSDS500. The boundary strength was produced by computing the gradient magnitude from $x$ and $y$ sobel filtering. 


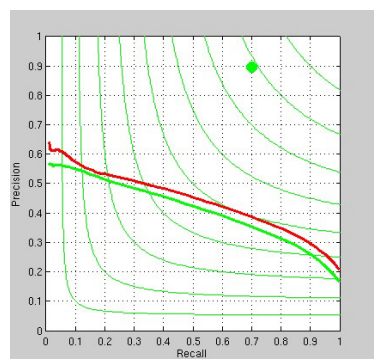

(a) Boundary strength

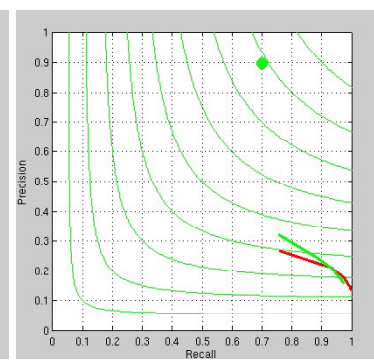

(b) Watershed
Figure 9. Precision and recall assessment

Figure 9 (b) shows a watershed precision and recall comparison between this work outcome (green line) and the conventional Matlab watershed implementation (red line) using BSDS500 assessment methodology against the train dataset.

The over segmentation image procedure allow to group 1000000 pixels into 201143 super pixels.

\subsection{Classification}

Obtained classification images for defined scenarios and their assessment outcomes are described in the following. Table 1 to Table 6 rows correspond to ground truth and columns (in the same order) to prediction.

Figure 10 shows classified images obtained only based on three spectral bands (scenario 1).

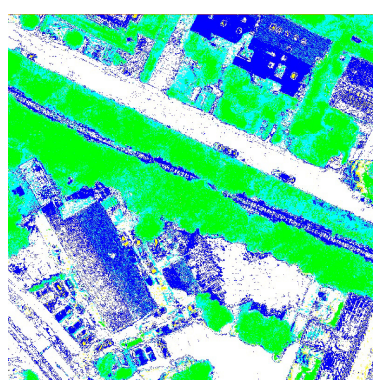

(a) Normal pixels

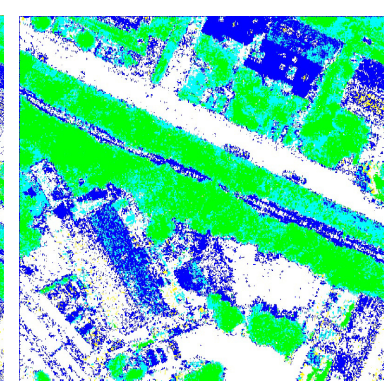

(b) Superpixels
Figure 10. Only spectral band classification images

Table 1 and Table 2 show confusion matrices for random forest pixel and superpixel (respectively) classification for the multispectral feature space.

\begin{tabular}{|l|r|r|r|r|r|}
\hline Category & \multicolumn{5}{|c|}{ Prediction(\%) } \\
\hline Building & 55.3 & 31.8 & 0.7 & 11.0 & 1.3 \\
Impervious surfaces & 14.4 & 72.8 & 3.2 & 8.6 & 1.1 \\
Tree & 1.4 & 1.6 & 72.1 & 24.9 & 0.2 \\
Low vegetation & 19.6 & 15.1 & 25.3 & 39.9 & 0.2 \\
Car & 42.7 & 38.5 & 2.2 & 9.6 & 7.1 \\
\hline
\end{tabular}

Table 1. Pixel based spectral feature space classification confusion matrix

\begin{tabular}{|l|r|r|r|r|r|}
\hline Category & \multicolumn{5}{|c|}{ Prediction(\%) } \\
\hline Building & 55.3 & 34.1 & 0.6 & 11.4 & 1.6 \\
Impervious surfaces & 15.0 & 72.8 & 3.0 & 8.2 & 1.0 \\
Tree & 1.8 & 1.6 & 71.7 & 24.8 & 0.1 \\
Low vegetation & 20.8 & 15.8 & 24.8 & 38.6 & 0.1 \\
Car & 42.7 & 41.3 & 1.8 & 8.6 & 5.6 \\
\hline
\end{tabular}

Table 2. Superpixel based spectral feature space classification confusion matrix

Figure 11 shows classified images based on a feature space composed by three spectral bands and the texture image channels (scenario 2).

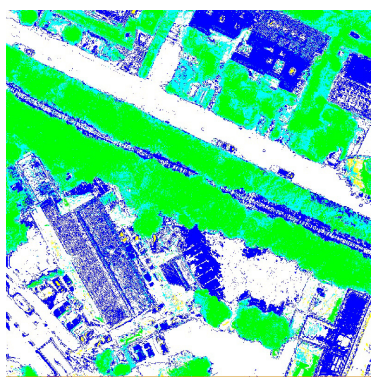

(a) Normal pixels

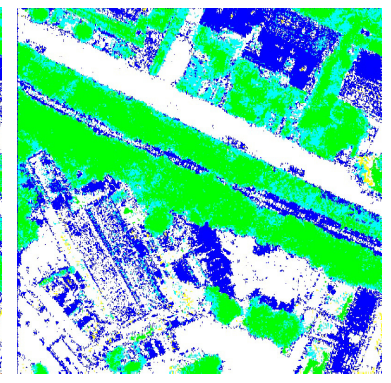

(b) Superpixels
Figure 11. Extended by texture feature space classification images

Table 3 and Table 4 show confusion matrices for random forest pixel and superpixel (respectively) classification for the feature space extended with channels for texture feature.

\begin{tabular}{|l|r|r|r|r|r|}
\hline Category & \multicolumn{5}{|c|}{ Prediction(\%) } \\
\hline Building & 57.3 & 34.7 & 0.8 & 6.5 & 0.7 \\
Impervious surfaces & 14.6 & 74.1 & 3.3 & 7.3 & 0.8 \\
Tree & 1.8 & 1.2 & 77.3 & 19.3 & 0.0 \\
Low vegetation & 19.5 & 15.1 & 26.6 & 38.7 & 0.1 \\
Car & 40.2 & 46.1 .6 & 2.4 & 6.6 & 4.7 \\
\hline
\end{tabular}

Table 3. Pixel based texture extended feature space classification confusion matrix

\begin{tabular}{|l|r|r|r|r|r|}
\hline Category & \multicolumn{5}{|c|}{ Prediction(\%) } \\
\hline Building & 53.8 & 38.5 & 0.8 & 6.2 & 0.8 \\
Impervious surfaces & 14.6 & 74.5 & 3.1 & 7.1 & 0.7 \\
Tree & 2.1 & 1.2 & 76.9 & 19.8 & 0.0 \\
Low vegetation & 19.8 & 15.9 & 25.1 & 39.0 & 0.1 \\
Car & 40.1 & 48.3 & 1.8 & 5.9 & 3.8 \\
\hline
\end{tabular}

Table 4. Superpixel based texture extended feature space classification confusion matrix

Figure 12 shows classified images based on a feature space composed by three spectral bands, texture image channels, NDVI channel and DSM channel (scenario 3). 


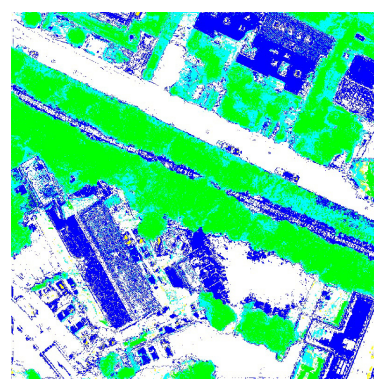

(a) Normal pixels

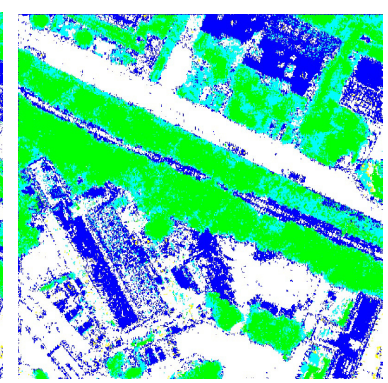

(b) Superpixels
Figure 12. Extended by texture, NDVI and DSM feature space classification images

Table 5 and Table 6 show confusion matrices for random forest pixel and superpixel (respectively) classification for the feature space extended with channels for texture, NDVI and DSM features.

\begin{tabular}{|l|r|r|r|r|r|}
\hline Category & \multicolumn{5}{|c|}{ Prediction(\%) } \\
\hline Building & 61.3 & 30.7 & 0.9 & 6.6 & 0.4 \\
Impervious surfaces & 13.3 & 74.6 & 3.3 & 8.2 & 0.5 \\
Tree & 2.0 & 1.0 & 76.3 & 20.7 & 0.0 \\
Low vegetation & 19.5 & 15.6 & 24.6 & 40.2 & 0.1 \\
Car & 39.6 & 46.6 & 2.1 & 7.7 & 4.1 \\
\hline
\end{tabular}

Table 5. Pixel based texture, NDVI and DSM extended feature space classification confusion matrix

\begin{tabular}{|l|r|r|r|r|r|}
\hline Category & \multicolumn{5}{|c|}{ Prediction(\%) } \\
\hline Building & 59.8 & 32.0 & 0.9 & 6.7 & 0.6 \\
Impervious surfaces & 13.9 & 74.6 & 3.0 & 8.1 & 0.5 \\
Tree & 2.1 & 1.0 & 75.6 & 21.4 & 0.0 \\
Low vegetation & 20.2 & 15.6 & 23.8 & 40.3 & 0.1 \\
Car & 39.2 & 48.7 & 1.7 & 7.1 & 3.3 \\
\hline
\end{tabular}

Table 6. Superpixel based texture, NDVI and DSM extended feature space classification confusion matrix

From confusion matrices the average classification accuracy (ACA) shown in Table 7 was calculated.

\begin{tabular}{|r|r|r|r|r|r|}
\hline \multicolumn{2}{|c|}{ Scenario1 } & \multicolumn{2}{c|}{ Scenario 2 } & \multicolumn{2}{c|}{ Scenario 3 } \\
\hline Pixel & Superpix & Pixel & Superpix & Pixel & Superpix \\
\hline 64.1 & 63.3 & 63.0 & 66.4 & 65.8 & 67.0 \\
\hline
\end{tabular}

Table 7. Overall accuracy

Precision and recall were also calculated for defined scenarios and respective outcomes are shown in Tables 8 and 9

\begin{tabular}{|l|r|r|r|r|r|r|}
\hline Category & \multicolumn{2}{|c|}{ Scenario1 } & \multicolumn{2}{c|}{ Scenario 2 } & \multicolumn{2}{c|}{ Scenario 3 } \\
\hline & Pixel & Super & Pixel & Super & Pixel & Super \\
Building & 50.3 & 47.7 & 50.9 & 49.0 & 53.9 & 52.4 \\
Impervious & 76.5 & 75.3 & 75.5 & 74.1 & 77.0 & 76.4 \\
surfaces & & & & & & \\
Tree & 84.4 & 84.8 & 84.8 & 85.4 & 85.1 & 85.8 \\
Low veg- & 23.8 & 23.3 & 28.3 & 28.5 & 27.4 & 27.2 \\
etation & & & & & & \\
Car & 25.3 & 21.5 & 25.8 & 22.6 & 30.2 & 27.3 \\
\hline
\end{tabular}

Table 8. Precision

\begin{tabular}{|l|r|r|r|r|r|r|}
\hline Category & \multicolumn{2}{|c|}{ Scenario1 } & \multicolumn{2}{c|}{ Scenario 2 } & \multicolumn{2}{c|}{ Scenario 3 } \\
\hline & Pixel & Super & Pixel & Super & Pixel & Super \\
Building & 55.3 & 52.3 & 57.3 & 53.8 & 61.3 & 59.8 \\
Impervious & 72.8 & 72.8 & 74.1 & 74.5 & 74.6 & 74.6 \\
surfaces & & & & & & \\
Tree & 72.1 & 71.8 & 77.7 & 76.9 & 76.3 & 75.6 \\
Low veg- & 39.9 & 38.6 & 38.7 & 39.0 & 40.2 & 40.3 \\
etation & & & & & & \\
Car & 7.1 & 5.6 & 4.7 & 3.8 & 4.0 & 3.3 \\
\hline
\end{tabular}

Table 9. Recall

\section{DISCUSSION}

As shown in Figure 9(a), $\frac{1}{3}$ of data used by a conventional convolution algorithm, it was possible to obtain similar sobel filter outcome. Figure 9(b) shows that Cartesian complex watershed implementation was as good as Matlab so it was appropriate for this study. Both implementations follow the same procedure described in section 2.3; the only difference is that CCLAI implementation uses edgels available in the underlying cartesian complex space.

It is possible to notice that when additional criteria are included in the feature space, the texture image first (Figure 11) and then the normalized difference vegetation index (NDVI) and SDM (Figure 12), the classified images get improved with respect to the classification obtained based only on the three spectral bands (Figure 11).

When comparing the respective classification images for pixels and superpixels in a same scenario, it is possible to notice how boundary superpixels have a rough finish and how extending the inside out and the outside in. This effect is due to the current implementation of the watershed transform and prevents the results for superpixels from being better. Despite the above, confusion matrices Table 1 / Table 2, Table 3 / Table 4 and Table 5 / Table 6 pairwise comparatives show that although creating the super pixels based on the watershed transform significantly decreased the number of elements of the space to be classified, the overall accuracy remained, so the performance gain by having to classify fewer elements did not mean a sacrifice of accuracy. It was better to have to classify 201143 superpixels than 1000000 pixels as the overall accuracy was kept.

Table 3 shows how including texture feature in the classification raised the correctly classified pixel percentage except for low vegetation and car categories in the case of pixel classification and 4 and Building and Car in the case of superpixels. This effect was stronger when NDVI and DSM features were included as shown in Figure 12 and Table 5 except for Car category. 
The overall accuracy always was below 70 percent but it should be considered that the assessment stage was conducted against the whole ground truth window (10000000 pixels).

As shown in Table 7 the use of a extended feature space with texture, NDVI and DSM data did not produce a significant effect in overall accuracy. However, Scenario 1 column, which did not included texture feature, shows how including superpixels and texture feature the overall accuracy decreases while Scenario 2 and Scenario 3 show how by including it increases overall accuracy.

Table 8 allows observing how, except for Impervious surfaces, the texture feature inclusion (scenario 2) slightly improves the precision regarding scenario 1.This tendency is kept with NDVI and SDM features inclusion which can be evidenced in scenario 3 with precision values higher for all categories.

Recall shows a similar effect in the case of scenario 3 (see Table 9) except for Car category, and except for Low vegetaton and Car categories in the case of scenario 2.

The inclusion of texture features taking into account geometric features in three different scales, starting at the most detailed possible, was key to improve the final classification results. This supports the argument that texture analysis is essential in the process of classification because it can provide an additional criterion to avoid erroneously mixing or separating adjacent space elements.

The use of watershed transform based on gradient texture favoured the proper definition of super pixels which, although it is true that it did not improve overall accuracy classification it did manage to preserve, with the added benefit of having a number of elements significantly reduced in the geometric space. This means that consequently the processing is significantly improved regardless of the classification method used.

It was possible to establish that the potential of superpixels in the classification is conditional on the simultaneous use of texture analysis. This suggests that processes not considering classifications texture analysis could reconsider the use of superpixels. Now, this study shows that it is necessary to improve the watershed transform in order to get better outcomes.

\section{CONCLUSIONS}

Sobel implementation based on edgels (1-cells) allows improving the processing independently of the algorithm used as it uses filter sizes of $2 \times 3$ and $3 \times 2$. This means that it uses a third less of the data than a conventional space based implementation using two $3 \times 3$ sized filters. In addition, the result is associated with a one-dimensional element as should happen from the topological point of view.

The availability of a underlying cartesian complex space provides a topologically correct oriented gradient calculation. In a conventional space when a disk is defined it is recommended not using the pixel linear arrangement which meets the diameter along the orientation as it does not lay in any of two sides but in the orientation. However, in a complex Cartesian space, there are interpixel elements that allow representing this situation properly by modelling that dividing line based on edgels and not on pixels.

The use of oriented matroids and their hyperplane central arrangement allows to improve the oriented gradient computation as the respective histograms are calculated once by sector and, at the final calculation, combined in agreement with each one of the specific orientations. The histogram calculation does not have to be done as many times as the number of orientations but it is conducted once and combined as many times as the number of orientations.

The benefits in processing and guaranteeing the topological model expressed in the previous paragraphs and although the results of the experiments show that the benefits of the inclusion of an underlying space based on Cartesian complex at the moment are very small, are evidence that it is worth continuing with the research.

As this study did not demonstrate improvement of thematic accuracy when using the proposed alternative space for land cover classification, in near future it will be investigated whether a complete implementation of a computational framework based on Cartesian complexes is able to accomplish such a challenge.

\section{ACKNOWLEDGEMENTS}

The Vaihingen data set was provided by the German Society for Photogrammetry, Remote Sensing and Geoinformation (DGPF) (Cramer, 2010).

Cristian Dallos wrote derivative filters, watershed and k-means first version for CCLAI.

Centro de Computo de Alto Desempeo of Universidad Distrital (CECAD) provided the matlab license and a Open SUSE Linux virtual machine for deployment.

\section{REFERENCES}

Arbelaez, P., Maire, M., Fowlkes, C. and Malik, J., 2011. Contour detection and hierarchical image segmentation. IEEE Trans. Pattern Anal. Mach. Intell. 33(5), pp. 898-916.

Canny, J., 1986. A computational approach to edge detection. IEEE Transactions on Pattern Analysis and Machine Intelligence PAMI-8(6), pp. 679-698.

Chen, C., Freedman, D. and Lampert, C. H., 2011. Enforcing topological constraints in random field image segmentation. In: Computer Vision and Pattern Recognition (CVPR), 2011 IEEE Conference on, pp. 2089-2096.

Cramer, M., 2010. The dgpf-test on digital airborne camera evaluation overview and test design. http://www.ifp.unistuttgart.de/dgpf/DKEP-Allg.html.

de Berg, M., Cheong, O., van Kreveld, M. and Overmars, M., 2008. Computational Geometry. Third edn, Springer Berlin Heidelberg.

Felzenszwalb, P. F. and Huttenlocher, D. P., 2004. Efficient graph-based image segmentation. Int. J. Comput. Vision 59(2), pp. 167-181.

Johansen, K., Coops, N. C., Gergel, S. E. and Stange, Y., 2007. Application of high spatial resolution satellite imagery for riparian and forest ecosystem classification. Remote Sensing of Environment $110(1)$, pp. $29-44$

Kanungo, T., Mount, D. M., Netanyahu, N. S., Piatko, C. D., Silverman, R. and Wu, A. Y., 2002. An efficient k-means clustering algorithm: analysis and implementation. IEEE Transactions on Pattern Analysis and Machine Intelligence 24(7), pp. 881-892.

Kong, C., Xu, K. and Wu, C., 2006. Classification and extraction of urban land-use information from high-resolution image based on object multi-features. Journal of China University of Geosciences 17(2), pp. $151-157$. 
Kong, T. and Rosenfeld, A., 1991. Digital topology: a comparison of the graph-based and topological approaches. g.m. reed, a.w. roscoe, r.f. wachter (eds.), topology and category theory in computer science, clarendon press, oxford, great britain. pp. 273287.

Kovalevsky, V., 2005. Algorithms in Digital Geometry Based on Cellular Topology. Springer Berlin Heidelberg, Berlin, Heidelberg, pp. 366-393.

Kovalevsky, V. A., 1989. Finite topology as applied to image analysis. In: Computer Vision, Graphics, and Image Processing, pp. 141-161.

Letscher, D. and Fritts, J., 2007. Image Segmentation Using Topological Persistence. Springer Berlin Heidelberg, Berlin, Heidelberg, pp. 587-595.

Leung, T. K. and Malik, J., 1996. Detecting, localizing and grouping repeated scene elements from an image. In: Proceedings of the 4th European Conference on Computer Vision-Volume I - Volume I, ECCV '96, Springer-Verlag, London, UK, UK, pp. 546-555.

Li, C. and Sun, Y., 2010. Active image: A shape and topology preserving segmentation method using b-spline free form deformations. In: 2010 IEEE International Conference on Image Processing, pp. 2221-2224.

Martin, D., Fowlkes, C., Tal, D. and Malik, J., 2001. A database of human segmented natural images and its application to evaluating segmentation algorithms and measuring ecological statistics. In: Proc. 8th Int'l Conf. Computer Vision, Vol. 2, pp. 416-423.

Munkres, J., 1999. Topology; a First Course. 2nd edition edn, Prentice-Hall.

Nunez-Iglesias, J., Kennedy, R., Parag, T., Shi, J. and Chklovskii, D. B., 2013. Machine learning of hierarchical clustering to segment $2 \mathrm{~d}$ and $3 \mathrm{~d}$ images. PLoS ONE 8(8), pp. 1-11.

Oxley, J. G., 2006. Matroid Theory (Oxford Graduate Texts in Mathematics). Oxford University Press, Inc., New York, NY, USA.

Rosenfeld, A., 1970. Connectivity in digital pictures. J. ACM 17(1), pp. 146-160.

Szeliski, R., 2010. Computer Vision: Algorithms and Applications. 1st edn, Springer-Verlag New York, Inc., New York, NY, USA.

Tso, B. and Mather, P., 2009. Classification Methods for Remotely Sensed Data. Environmental engineering, CRC Press, Taylor \& Francis Group.

Vincent, L. and Soille, P., 1991. Watersheds in digital spaces: an efficient algorithm based on immersion simulations. IEEE Transactions on Pattern Analysis and Machine Intelligence 13(6), pp. 583-598.

Whitney, H., 1935. On the abstract properties of linear dependence. American Journal of Mathematics 57, pp. 509-533.

Worboys, M. and Duckham, M., 2004. GIS: A Computing Perspective, 2Nd Edition. CRC Press, Inc., Boca Raton, FL, USA.

Yu, Q., Gong, P., Clinton, N., Biging, G., Kelly, M. and Schirokauer, D., 2006. Object-based detailed vegetation classification with airborne high spatial resolution remote sensing imagery. Photogrammetric Engineering \& Remote Sensing 72(7), pp. 799 811. 